\title{
Monitoring Medicine Intake in the Networked Home: The iCabiNET Solution
}

\author{
Martín López-Nores, José J. Pazos-Arias, Jorge García-Duque and Yolanda Blanco-Fernández \\ Department of Telematics Engineering, University of Vigo (Spain) \\ Email: \{mlnores,jose,jgd,yolanda\}@ det.uvigo.es
}

\begin{abstract}
We introduce an intelligent medicine cabinet as a new element of a residential network, acting as a secure place to store sensitive health information, and therefrom access a range of interactive health care applications. This paper describes the functionalities related to monitoring the intake of prescription and over-the-counter drugs, harnessing recent advances in smart medicine packaging and home networking. Compared to previous systems, ours helps reducing the risk of medicine misuse, featuring higher precision and enhanced interactive facilities that reach in and out of home. This contributes to solving a problem that impinges heavily on the well-being of people and the economics of public health systems.
\end{abstract}

\section{INTRODUCTION}

The Telematics Engineering Department of the University of Vigo is working on a prototype intelligent medicine cabinet (hereafter referred to as the iCabiNET), that will integrate various functionalities related to health care informatics within a consumer device. Specifically, this system will act as (i) a smart medicine manager, (ii) a secure place to store sensitive health-related information, and (iii) a gateway to access remote health care applications.

In this paper, we shall focus on the concept of smart medicine manager. The basic idea is to automatically monitor the intake of prescription and over-the-counter drugs, for example, to warn the user when it is time to take a pill, to keep watch over drug availability for the next few days, to check expiration dates, etc. Some precedents can be found in [2], [5], [7], [10], with systems that employed RFID devices to recognize medicines, plus weighing scales to guess the doses available. This approach has turned out to be ineffective, due to the following drawbacks:

- To function properly, the weighing scales require the user to pick one medicament at a time and put it back before picking another. This may be a cumbersome discipline to follow in many cases, and any oblivion can lead to monitoring imprecision.

- Noticeably, the previous systems have very limited possibilities to reach the users, merely using embedded screens, lights or alarms. This way, there is a clear risk of medicine misuse if the user happens to be out of home, or simply in a room where he/she cannot see the lights or hear the alarms.

- Finally, the previous systems rely on the assumption that people use a medicine cabinet as the only place for medication keeping. Nevertheless, polling data reveals that this may not be true in as many as $90 \%$ of the cases, because people tend to store their medicines in various places around the house [4].

The iCabiNET system tackles the aforementioned problems by looking at recent advances in the fields of residential networks [1] and smart medicine packaging [6]. The following sections describe how we combine these technologies, plus a couple of usage scenarios to illustrate the benefits and the potential uses of our approach. Some details about a prototype implementation are provided before the ending conclusions.

\section{SYSTEM OVERVIEW}

The first notable feature of the iCabiNET is that it allows monitoring medicine intake from anywhere, whereas previous systems required the user to be in front of the weighing scales. This advantage arises from the smart packaging technologies currently promoted by stakeholders of the pharmaceutical industry, to integrate both RFIDs and sensors with the packaging of the medicines. ${ }^{1}$ This allows tracking drug names and doses available with the highest precision and no risk of mistaking one drug for another. Knowing that, we have designed the iCabiNET as an in-home appliance connected to a residential network, through which it polls RFID readers deployed around the house (the RR devices in Fig. 1).

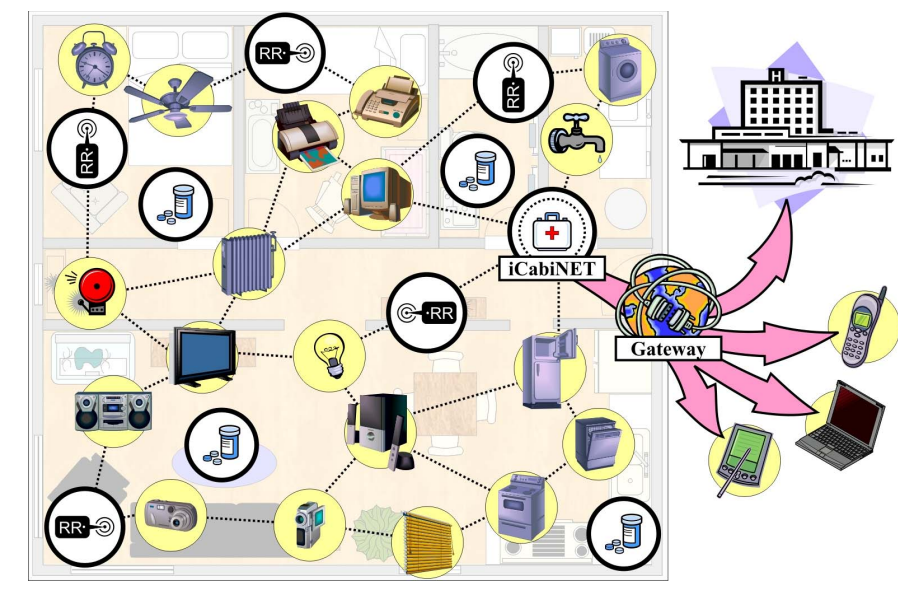

Fig. 1. The iCabiNET system in a residential network.

With the information gathered about available medicines and doses, the iCabiNET delivers functionality driven by

\footnotetext{
${ }^{1}$ The most common example of smart packaging is that of smart blister packs, equipped with sensors that record the removal of a pill simply by breaking an electric flow into the RFID's integrated circuit.
} 
operation flows that describe sequences of actions related to the use of specific drugs. These actions may have to do with medication guidelines (e.g. "the user should take one of these pills every 4 to 6 hours"), conditions related to patient characteristics (e.g. age or gender), or actions to perform in the events of oblivion (e.g. "wait up to 90 minutes before warning the user"), expiration (e.g. "deliver increasingly serious warnings day after day"), nearing depletion (e.g. "arrange a meeting with the doctor to get a new prescription"), discontinuation (e.g. "restart the medication at a lower dose"), abuse (e.g. "warn the local authorities"), etc. Some operation flows are preloaded in the iCabiNET for common processes, while others can be entered by the users or downloaded from external sources (e.g. health institutions or pharmaceutical companies). In any case, the flows can be valid for anyone or tailored for a given individual.

The point that makes the iCabiNET most advantageous with regard to previous systems is that, being connected to a residential network, it has limitless possibilities to reach the users. To this aim, we have extended the semantic composition mechanisms of [3] to instantiate the generic actions in the operation flows using any of the devices available in the house, and taking into account the user's context (sleeping, out of home, watching TV, etc). Thus, for example, a "warn the user" action can be automatically made to trigger an alarm clock, to interrupt a TV program, to make a phone call, etc. Moreover, the plug-and-play features of the residential network standards allow incorporating new devices with, at the most, a simple update of the iCabiNET's software.

\section{USAGE SCENARIOS}

Scenario \#1: Ann is having breakfast before going to work, and switches on the radio to hear the first news of the day. When she is about to turn off the apparatus, the iCabiNET reminds her of the medicines she should carry, playing a prerecorded message. Later, following the prescription issued by the doctor - loaded into the iCabiNET directly from the health center- Ann receives an SMS message in her mobile phone every three hours to remind taking her drugs. At the end of the day, when Ann is back in her house, the iCabiNET attempts to check that the available doses have decreased as expected, but it turns out that Ann has left them behind. In this case, the iCabiNET rings the in-home telephone to ask Ann whether she has taken the medicines correctly; she replies affirmatively by pressing the asterisk key. During the night, the alarm clock in Ann's bedroom will be responsible for waking her up when it is time for new doses.

Scenario \#2: While having a walk around the city, Bob decides to buy a certain over-the-counter drug to treat his allergy to pollen. Afterwards, when he enters his house, the iCabiNET records the tablets he has bought, and automatically downloads generic medication guidelines from the manufacturer. A few days later, Bob is watching TV in the living room. When it is time to take a pill, the iCabiNET pops up a reminder on the screen, indicating the drug's commercial name, a photograph of its packaging and the recommended dose. For the best comfort, the system starts flashing the lights of the room where Bob had left the tablets the last time he took one. As Bob takes a new dose, the iCabiNET finds that the pills are running out; so, when he sits back in the sofa, he is faced with an interactive TV application that he can use to buy new supplies from an online drugstore. Bob uses the remote control to enter shipping and payment details, and takes the opportunity to buy some throat lozenges he likes.

\section{PROTOTYPE IMPLEMENTATION}

We have a working prototype of the iCabiNET system, implemented over a Bluetooth-enabled mobile phone that can be connected to OSGi-based residential networks [9]. A J2ME virtual machine runs the algorithms that instantiate the generic actions of the operation flows - expressed in the BPEL language [8] — using any devices available, completely regardless of particular vendors and manufacturers.

In our trials, we used purpose-built smart blister packs and RFID readers, because smart packaging technology is not yet available in retail drugs. We have confirmed that the prototype is capable of realizing the scenarios of the preceding section.

\section{CONClusion}

Medicine misuse is a growing problem, with a severe impact on the well-being of people and the economics of public health systems. The iCabiNET system exploits recent advances in smart medicine packaging to provide the greatest flexibility and precision in monitoring prescription and overthe-counter drugs, leaning on home networking solutions to reach the users through whichever technological means become available. Thus, its functionalities as a smart medicine manager go far beyond those of previous systems, including unprecedented capabilities to interact with health institutions, drug manufacturers and retailers.

\section{ACKNOWLEDGMENT}

This work has been partially funded by the Spanish Ministry of Education and Science through Project TSI2007-61599.

\section{REFERENCES}

[1] L. Baxter. Residential networks. Thomson Delmar Learning, 2005.

[2] N. Bricon-Souf and C. Newman. Context awareness in health care: A review. Intl. J. of Medical Inform., 76(1):2-12, 2007.

[3] R. P. Díaz-Redondo, A. Fernández-Vilas, M. Ramos-Cabrer, J. J. PazosArias, and M. Rey-López. Enhancing residential gateways: OSGi service composition. IEEE Trans. Consumer Electron., 53(1):87-95, 2007.

[4] K. Fishkin and S. Consolvo. Results of medication questionnaire \#1. Technical Report IRS-TR-03-002, Intel Research, 2003.

[5] C. Floerkemeier and F. Siegemund. Improving the effectiveness of medical treatment with pervasive computing technologies. In Proc. of Ubicomp Workshops, Seattle, USA, October 2003.

[6] P. Harrop. Opportunities in pharmaceutical RFID and smart packaging. IDTechEx, 2006.

[7] L. Ho, M. Moh, Z. Walker, T. Hamada, and C.-F. Su. A prototype on RFID and sensor networks for elder healthcare. In Proc. of SIGCOMM Workshops, Philadelphia, USA, August 2005.

[8] M. Juric. Business Process Execution Language for Web Services. Packt Publishing, 2006.

[9] OSGi Alliance. Open Service Gateway initiative. http://www.osgi.org.

[10] D. Wan. Magic Medicine Cabinet: A situated portal for consumer healthcare. In Proc. 1st International Symp. on Handheld and Ubiquitous Computing, Karlsruhe, Germany, September 1999. 